\title{
Disentangling the gamma-ray emission of NGC 1275 and that of the Perseus cluster
}

\author{
S. Colafrancesco ${ }^{1,2}$, P. Marchegiani ${ }^{2,3}$, and P. Giommi ${ }^{1}$ \\ 1 ASI-ASDC c/o ESRIN, via G. Galilei snc, 00040 Frascati, Italy \\ e-mail: colafrancesco@asdc.asi.it \\ 2 INAF - Osservatorio Astronomico di Roma via Frascati 33, 00040 Monteporzio, Italy \\ e-mail: sergio.colafrancesco@oa-roma.inaf.it \\ 3 Dipartimento di Fisica, Università di Roma La Sapienza, P.le A. Moro 2, Roma, Italy \\ e-mail: paolo.marchegiani@oa-roma.inaf.it
}

Received 9 March 2010 / Accepted 10 June 2010

ABSTRACT

\begin{abstract}
Context. The Gamma-ray emission from galaxy clusters hosting active galaxies is a complex combination of diffuse and point-like emission features with different spectral and spatial properties.

Aims. We discuss in details the case of the Perseus cluster containing the radio-galaxy NGC 1275 that has been recently detected as a bright gamma-ray source by the Fermi-LAT experiment, in order to disentangle the sources of emission.

Methods. We provide a detailed study of the gamma-ray emission coming from the core of Perseus by modeling the central AGN emission with a multiple plasma blob model, and the emission from the extended cluster atmosphere with both a warming ray (WR) model and dark matter (DM) neutralino annihilation models. We set constraints on both the central galaxy and cluster SED models by using both archival multi-frequency data and the recent very high energy observations obtained by Fermi and MAGIC.

Results. We find that: i) in all the viable models for the cluster gamma-ray emission, the emission detected recently by Fermi from the center of the Perseus cluster is dominated by the active galaxy NGC 1275, that is found in a high-emission state; ii) the diffuse gamma-ray emission of the cluster, in the WR model and in the DM models with the highest allowed normalization, could be detected by Fermi if the central emission from NGC 1275 is in a low-emission state; iii) Fermi can have the possibility to resolve and detect the diffuse gamma-ray flux (predicted by the WR model) coming from the outer corona of the Perseus cluster atmosphere at distances $r \gtrsim 800 \mathrm{kpc}$. These results are consistent with the evidence that in the other frequency bands, the diffuse cluster emission dominates on the central galaxy one at low radio frequencies with $v \lesssim 1 \mathrm{GHz}$ and at $\mathrm{X}$-ray energies of order of $E \sim \mathrm{keV}$.

Conclusions. Our results show that a simultaneous study of the various emission mechanisms that produce diffuse gamma-rays from galaxy clusters and the study of the emission mechanisms that produce gamma-rays from active galaxies residing in the cluster atmospheres is absolutely crucial first to disentangle the spectral and spatial characteristics of the gamma-ray emission and secondly to assess the optimal observational strategy in the attempt to reveal the still elusive diffuse gamma-ray emission widely predicted for the atmospheres of large-scale structures.
\end{abstract}

Key words. galaxies: clusters: general - galaxies: clusters: individual: Perseus - gamma rays: galaxies: clusters - dark matter cosmology: theory

\section{Introduction}

The radio-galaxy NGC 1275 (3C 84) has been recently detected by Fermi as a source of high-energy gamma rays with an average flux and power-law photon index of $F(>100 \mathrm{MeV})=$ $(2.10 \pm 0.23) \times 10^{-7} \mathrm{~cm}^{-2} \mathrm{~s}^{-1}$ and $\gamma=2.17 \pm 0.05$, respectively (Abdo et al. 2009). The emission detected by the FermiLAT is consistent with a point source centered at the nucleus of NGC 1275. The gamma-ray flux measured with Fermi-LAT is almost an order-of-magnitude brighter than the EGRET flux upper limit, $F(>100 \mathrm{MeV})<3.72 \times 10^{-8} \mathrm{~cm}^{-2} \mathrm{~s}^{-1}$ (Reimer et al. 2003), and therefore implies that NGC 1275 is varying significantly at gamma-rays on time scales from months to years (see discussion in Abdo et al. 2009).

The Fermi results on NGC 1275 have clearly an impact on the models for the possible gamma-ray emission originating from the surrounding Perseus cluster atmosphere because they limit the detectability of the amount and of the spectral energy distribution (SED) of the diffuse gamma-ray emission that can originate from the hosting galaxy cluster.
The Perseus cluster (as many other galaxy clusters) is indeed expected to be a source of gamma-ray emission due to various emission mechanisms (see e.g., Colafrancesco 2007-2009, for a recent discussion): i) inverse Compton scattering (ICS) of CMB photons and relativistic bremsstrahlung of primary cosmic ray particles (mainly electrons) (see, e.g., Houston et al. 1984; Sarazin 1999; Miniati et al. 2001; Brunetti 2003; Colafrancesco et al. 2005); ii) neutral pion e.m. decay produced by pp collisions, and ICS of CMB photons and relativistic bremsstrahlung of secondary electrons produced in the same pp collisions (e.g., Dennison 1980; Colafrancesco \& Blasi 1998; Marchegiani et al. 2007); iii) neutral pion e.m. decay produced by warming rays (WR) interaction and ICS of CMB photons and relativistic bremsstrahlung of secondary electrons produced in the same pp collisions (Colafrancesco \& Marchegiani 2008, 2009); iv) neutral pion e.m. decay produced by neutralino dark matter annihilation, and ICS of CMB photons and relativistic bremsstrahlung of secondary electrons produced in the same DM annihilation processes (Colafrancesco \& Mele 2001; Colafrancesco et al. 2006, 2010); v) ICS and relativistic bremsstrahlung from PeV 
electron-positron pairs produced via interactions of ultra high energy photons (particles) with the CMB photons (Timokhin et al. 2004; Inoue et al. 2005).

Experimental limits on the gamma-ray emission from the Perseus cluster and its central galaxy NGC 1275 have been obtained by various experiments. Gamma-ray observations toward NGC 1275 and the Perseus clusters were first reported in the 1980's by Strong \& Bignami (1983). The COS B data, taken between 1975-1979 (Strong et al. 1982; Mayer-Hasselwander et al. 1982), show a gamma-ray excess at the position of the galaxy, although evidence for emission uniquely related to NGC 1275 is ambiguous (in fact positional uncertainties were not given for the COS B data). Interpreted as emission from NGC 1275, the gamma-ray flux is $F(>70 \mathrm{MeV})=8.3 \times$ $10^{-7} \mathrm{~cm}^{-2} \mathrm{~s}^{-1}$, which is more than an order of magnitude higher than the upper limit reported by EGRET. Beyond the Fermi detection and the EGRET upper limit already mentioned, recent limits on $\mathrm{TeV}$ emission at $E>400 \mathrm{GeV}$ have been obtained by Whipple $\left(F<7.4 \times 10^{-12} \mathrm{erg} \mathrm{cm}^{-2} \mathrm{~s}^{-1}\right.$; see Perkins et al. 2006 with a radial cut of $0.3 \mathrm{deg}$ and a spectral index of -2.1$)$ and from MAGIC at $E>100 \mathrm{GeV}(F<4.6$ to $7.5 \times 10^{-12} \mathrm{~cm}^{-2} \mathrm{~s}^{-1}$, for a point like source and spectral indices in the range -1.5 to -2.5 ; see Aleksic et al. 2010). Very recently, a new upper limit has been obtained with VERITAS (Acciari et al. 2009 for a point like source and a spectral index of -2.5), with $F(>188 \mathrm{GeV})<5.11 \times 10^{-12} \mathrm{~cm}^{-2} \mathrm{~s}^{-1}$ while NGC 1275 was in a quite low state at the gamma-ray energies probed by Fermi. We refer the reader to Aleksic et al. (2010) for a discussion of the comparison of the limits obtained by Cherenkov telescopes.

At soft gamma-ray energies, INTEGRAL observations of the Perseus cluster found no evidence for a diffuse power-law emission which would dominate the emission above $30 \mathrm{keV}$ (Eckert \& Paltani 2009). However, the angular resolution of IBIS/ISGRI is not sufficient to disentangle the point-like emission from the diffuse emission component, so it is not possible to set any definite upper limit on the diffuse non-thermal emission from the cluster.

At X-ray energies, Perseus is the nearest example of a prototypical cool core cluster in which the intra-cluster (IC) gas temperature decreases from the outer region value of $\sim 7 \mathrm{keV}$ down to the value of $\sim 3 \mathrm{keV}$ found at its center (see Colafrancesco \& Marchegiani 2008 and references therein for a recent discussion) where the cluster hosts the giant elliptical galaxy NGC 1275.

The Perseus cluster appears, moreover, to contain a nonthermal component, namely an excess of hard X-ray emission above the thermal bremsstrahlung from the diffuse hot cluster gas. Based on a deep Chandra observation, the non-thermal $\mathrm{X}$-ray component has been mapped over the core of the cluster and shows a morphology similar to the radio mini-halo (Sanders et al. 2005; Sanders \& Fabian 2007). Notice, however, that this claim was questioned on the basis of a long XMM-Newton exposure (Molendi \& Gastaldello 2009). Above $10 \mathrm{keV}$, a hard X-ray component has been detected with HEAO-1 (Primini et al. 1981) and BeppoSAX/PDS (Nevalainen et al. 2004), although it was not detected with CGRO/OSSE in the 0.05-10 MeV range (Osako et al. 1994). It is interesting to notice that Perseus is the only cluster - out of ten clusters detected in the $15-55 \mathrm{keV}$ range with Swift/BAT (Ajello et al. 2009) - that displays a high-energy non-thermal component up to $200 \mathrm{keV}$, even though the hard tail seen in the BAT spectrum is likely due to nuclear emission from NGC 1275 rather than to non-thermal emission from the cluster atmosphere. This idea is supported by possible flux variations compared to past hard X-ray observations, and by the fact that the extrapolation of the BAT spectrum is in good agreement with the luminosity of the nucleus as measured with XMM-Newton (Churazov et al. 2003).

This supports, once more, the interpretation that the nonthermal emission of hard X-rays and of the gamma-rays is produced by the central active galaxy.

NGC 1275 has been variously classified as a Seyfert 1.5 (because of its emission-line optical spectrum, where broad lines are detected, see Véron-Cetty \& Véron 1998), but also as a blazar (due to the strong and rapid variability of the continuum emission and its polarization, see e.g., Angel \& Stockman 1980; see also Pronik et al. 1999). The very bright radio source Perseus A (also known as 3C 84) found in NGC 1275 has a strong, compact nucleus which has been studied in detail with VLBI (Vermeulen et al. 1994; Taylor \& Vermeulen 1996; Walker et al. 2000; Asada et al. 2006) and a bowshock-like southern jet component (Kellermann et al. 2004; Lister et al. 2009). The radio emission continues on larger scales, and shows a clear interaction with the intra cluster (IC) gas. Observations with ROSAT (Boehringer et al. 1993) and later Chandra (Fabian et al. 2003a, 2006) reveal the presence of cavities in the IC gas, suggesting that the jets of 3C 84 have blown multiple bubbles in the atmosphere of the Perseus cluster. On even larger scales, Perseus exhibits a mini-halo of size $\sim 300 \mathrm{kpc}$, best seen at low-frequency radio emission (Burns 1990), that is likely produced by synchrotron emission from widely distributed relativistic particles and fields energized in the central regions of the cluster.

On the theoretical side, several predictions on the intensity and spectral properties of the Perseus cluster gamma-ray emission have been presented. Pfrommer (2008) predicted a total gamma-ray flux at $E>100 \mathrm{MeV}$ that is in the range $(3.2-5.6) \times$ $10^{-9} \mathrm{~cm}^{-2} \mathrm{~s}^{-1}$ depending on the details of their models of relativistic electrons accelerated at cosmological structure formation shocks and those that are produced in hadronic interactions of cosmic rays with ambient gas protons. Kushnir \& Waxman (2009) predicted, in a simple model that explains the HXR emission from galaxy clusters as ICS scattering of CMB photons by relativistic electrons accelerated at the accretion shock surrounding the cluster, a gamma-ray flux at $E>50 \mathrm{GeV}$ for Perseus (within $0.1 \mathrm{deg}$ ) of $3.1 \times 10^{-13} \mathrm{~cm}^{-2} \mathrm{~s}^{-1}$ for the pion decay channel production, and of $1.5 \times 10^{-13} \mathrm{~cm}^{-2} \mathrm{~s}^{-1}$ for the ICS channel production. Colafrancesco \& Marchegiani (2008) predicted that the simplest WR model should produced a total (maximal) gamma-ray flux for Perseus (integrated within its virial radius) of $1.2 \times 10^{-8} \mathrm{~cm}^{-2} \mathrm{~s}^{-1}$ for photons with $E>100 \mathrm{MeV}$.

The predictions of the various models have now to contend with the new observation of the central part of the Perseus cluster atmosphere where the NGC 1275 gamma-ray emission results dominant. Furthermore, the understanding of the nature of the central radio galaxy SED (and especially of its high-E branch at $E \gtrsim \mathrm{GeV}$ ) can help in setting more precise constraints on the amount of diffuse gamma-ray emission of the Perseus cluster, and on the SED of the cluster non-thermal emission models, and can help also in outlining the optimal strategy for the detectability of galaxy clusters at gamma-ray energies.

In this paper we study the gamma-ray emission of the composite system of the radio galaxy - cluster that have different origins and hence different spectral, temporal and spatial characteristics. We perform this study with the aim to disentangle the diffuse and point-like emissions by using a full multifrequency strategy. To this aim, we discuss in Sect. 2 the theory of gamma-ray emission in systems composed by a galaxy cluster plus a central AGN. In Sect. 3 we discuss a model for the multi-frequency SED of NGC 1275 that is able to recover the 
different sets of observations for this radio galaxy, from radio to high-energy gamma-rays and TeVs. Section 4 presents the predictions for the diffuse emission of the atmosphere of the Perseus cluster in the region surrounding NGC 1275 for three different physical models: i) the Warming Ray model, whose multi-frequency SED is based on its ability to reproduce the temperature profile of the hot intracluster gas; ii) three models for neutralino dark matter annihilation with different neutralino mass and composition; iii) a pure leptonic model with a powerlaw relativistic electron spectrum. In Sect. 5 we will discuss the comparison of the SEDs for NGC 1275 and for the surrounding Perseus cluster atmosphere using the largest possible frequency coverage, from radio to $\mathrm{TeV}$ energies. We will show that a full multi-frequency, and multi-component study, is able to disentangle the point-like vs. diffuse emissions of such complex astrophysical systems. The final Sect. 6 is devoted to the discussion of our results and to summarize our conclusions.

Throughout the paper, we use a flat, vacuum-dominated cosmological model with $\Omega_{\mathrm{m}}=0.3, \Omega_{\Lambda}=0.7$ and $h=0.7$.

\section{The SED of a cluster containing a central radio galaxy: setting the model}

The evidence so far discussed indicates that in order to fully understand the level and the spectral properties of the high-E emission from the center of the Perseus cluster, we need to model in details the two separate components, i.e. NGC 1275 and the Perseus cluster atmosphere.

The model we discuss here consists of a diffuse plasma component, associated to the atmosphere of the Perseus cluster, and a compact central component, mainly provided from the jets and blobs emitted from the NGC 1275 galaxy. We want to compare the two different SEDs in the same spatial region, i.e. in the central part of the cluster.

In this region we expect most of the diffuse emission produced by the cluster, since the thermal bremsstrahlung emissivity is $\propto n_{\text {th }}(r)^{2}$, the non-thermal cluster emission produced by secondary electrons is $\propto n_{\mathrm{th}} \times n_{\mathrm{CR}}$, and the DM-produced emission is $\propto n_{\mathrm{DM}}^{2}(r)$, usually peaked towards the cluster center (here $n_{\mathrm{th}}, n_{\mathrm{CR}}$ and $n_{\mathrm{DM}}$ are the number densities of thermal particles, cosmic rays and DM particles, respectively). To model the Perseus SED we consider, specifically, the WR model (Colafrancesco \& Marchegiani 2008), in which a population of relativistic protons heat the gas due to Coulomb and hadronic interactions bringing it to a quasi stationary cooling-heating equilibrium and, at the same time, produce via $\mathrm{p}-\mathrm{p}$ collisions secondary electrons and direct gamma-ray emission (via neutral pion decay). Contrary to standard secondary electron models (see, e.g., Marchegiani et al. 2007) in which the CR density (and hence the intensity of the produced radiation) is not known a priori, the CR density and its spatial distribution in the WR model are fixed by the condition of reproducing the cluster gas temperature distribution.

Dark matter annihilation in the central region of the cluster is also the dominant source of emission in neutralino DM models: therefore, we consider the SEDs predicted in a few neutralino DM models whose intensity is, however, uncertain due mainly to the unknown value of the annihilation cross-section (see, e.g., the discussion made by Colafrancesco \& Marchegiani 2009).

We also discuss the emission features of a pure leptonic model, in which a diffuse population of relativistic electrons produce $\gamma$-ray emission in the cluster center: this case will be used to set the maximum density of non-thermal electrons allowed by the gamma-ray upper limit measured by EGRET.

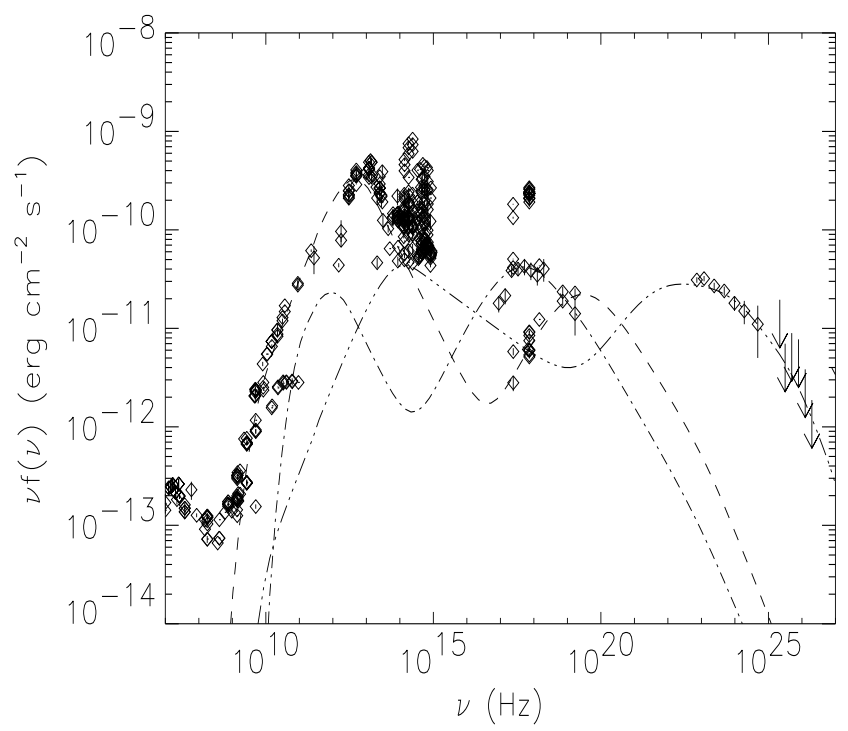

Fig. 1. The SED of NGC 1275 fitted by our CM model with three separated SSC components (see Table 1): component 1 (dashed), 2 (three dots - dashes) and 3 (dot-dash). Data are from NED, from Fermi (Abdo et al. 2009) and from MAGIC (Aleksic et al. 2010).

As for the active galaxy NGC 1275, we describe its SED as produced in a model (hereafter referred to as the Cannon Model or CM model) with multiple components (Colafrancesco et al. 2010, in prep.), finding the optimal jet-blob structure that is able to reproduce the observed multi-frequency SED. Specifically, we assume in our CM model that different blobs of relativistic plasma are emitted at different times from the central source (close to the hypothetical SMBH). This model predicts that the AGN SED is the combination of the SSC emissions of each separate blob and the ICS emission that each blob produces on the photons emitted from external radiation fields, including those emitted from the SSC part of each emitted blob. A more simplified version of this model has been already used to describe the SED of the blazar S05 0716+714 (see Giommi et al. 2008; Vittorini et al. 2009).

We describe, in the following, the specific SEDs of the NGC 1275 galaxy of the Perseus cluster separately and then the combination of the two SEDs in the same spatial region.

\section{The SED of NGC 1275}

Figure 1 shows the fit to the multi- $v$ SED of NGC 1275 obtained in the CM model (see Colafrancesco et al. 2010, in prep.) with the composition of three SSC components, whose parameters are reported in Table 1, generated by three separated plasma blobs blasted away from the inner regions of the NGC 1275 nucleus. The first blob of intermediate synchrotron peak (SP) frequency and larger radius produces a SSC emission that recovers the low $-v$ radio emission and the synchrotron peak and, in its ICS branch, also the historical X-ray data with lower flux. The second, high SP frequency and smaller blob produces a SSC emission that is systematically displaced towards high frequencies and fits the Fermi data while remaining subdominant at all other frequencies. The third, lowest SP frequency blob produces a SSC emission that is able to fit the X-ray data of higher flux with its IC peak emission but remains sub-dominant at all other frequencies. The three components are interpreted as emissions from blobs blasted away at different times so that their combination could explain also the source variability. Table 1 reports 
Table 1. Parameters of the three blobs of the CM model whose SEDs are shown in Fig.1.

\begin{tabular}{ccccccccc}
\hline \hline Component & $\log N\left(\mathrm{~cm}^{-3}\right)$ & $p_{1}$ & $p_{2}$ & $\log \gamma_{b}$ & $B(\mathrm{G})$ & $\delta$ & $r(\mathrm{pc})$ & $z$ \\
\hline 1 & -1.15 & 0.95 & 4.4 & 3.2 & 0.1 & 4.0 & 0.21 & 0.0179 \\
2 & 0.83 & 1.2 & 3.5 & 3.7 & 0.1 & 8.0 & 0.008 & 0.0179 \\
3 & 3.2 & 1.0 & 4.4 & 2.7 & 0.1 & 8.0 & 0.003 & 0.0179 \\
\hline
\end{tabular}

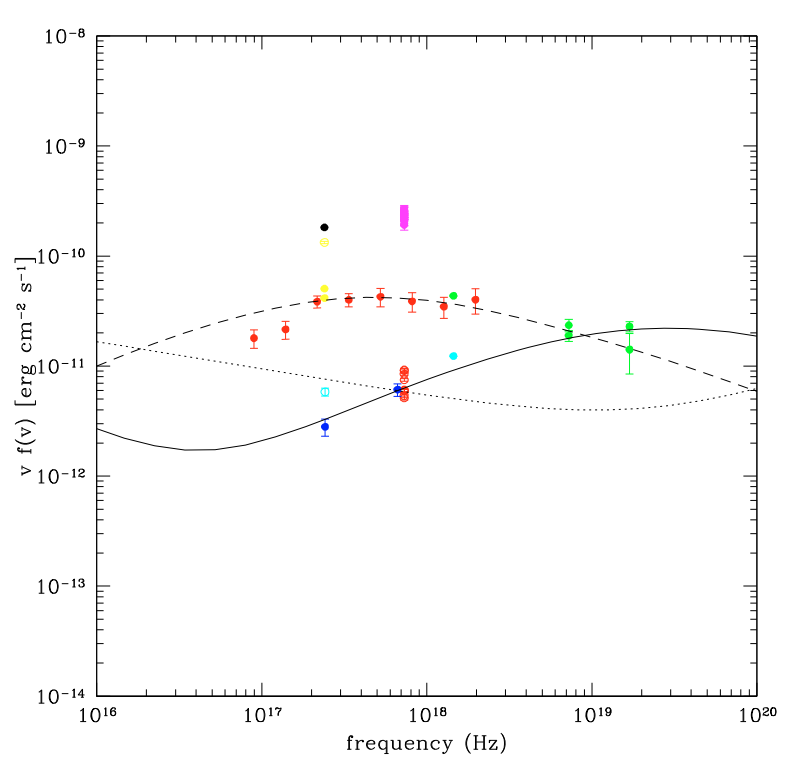

Fig. 2. The multiple-component SED of NGC 1275 in the UV to hard $\mathrm{X}$-ray frequency range. The three SED components have parameters listed in Table 1: component 1 (solid line), 2 (dotted line) and 3 (dashed line). Data are from: RASS (black), Einstein IPC (yellow), BeppoSAX WFC (magenta), Swift XRT (red), Chandra (blue), XMM (cyan), Integral (green).

the values of the boosting parameter $\delta=[\Gamma(1-\beta \cos \theta)]^{-1}$ (here $\beta=v / c$ is the bulk velocity of the jet, $\Gamma=\left(1-\beta^{2}\right)^{-1 / 2}$ and $\theta$ is the angle between the direction of the jet and the line of sight) that take values between 4 and 8 for the three blobs. These values are lower than the typical values of order of a few tens found for blazars, consistently with the fact that in the radio-galaxy NGC 1275 the jet is not oriented towards the observer.

If we assume, for the sake of discussion, a viewing angle $\theta \leq$ $1 / \delta_{\max }=0.125$, e.g., $\theta \sim 10^{-1} \mathrm{rad}$, the values of $\delta$ are compatible with values of the blob bulk velocity of $\Gamma \sim 2-6$, for which the emitted radiation is beamed at angles $\theta \simeq 1 / \Gamma=0.17-0.5$ $\mathrm{rad}$. A viewing angle of $0.1 \mathrm{rad}$ is hence inside the viewing cone within which the emission is beamed, even though this angle is larger and the bulk velocity is lower than the values typically found in blazars, in agreement with the fact that NGC 1275 is not a canonical blazar, but shows blobs ejected with a viewing angle (w.r.t. the line of sight) relatively large (but still not of order of $\sim 90 \mathrm{deg}$, as for prototypical radio galaxies) that hence determines emission characteristics intermediate between a radio galaxy nucleus and those of a blazar nucleus.

X-ray observations of NGC 1275 over a wide historical period show that the flux of this sources varied substantially (over almost two orders of magnitude) as well as its spectral shape, indicating the presence of different SED components.

The X-ray part of the SED of NGC 1275 (see Fig. 2) shows, in fact, that three blobs SEDs are required to fit the various sets of historical data accumulated for NGC 1275.
In the soft X-ray band, the lowest flux component includes Chandra data (Balmaverde et al. 2006, blue points), the SwiftXRT data of the lowest stage (data from ASDC; red empty dots), the XMM data that show a slightly higher flux with respect to the previous data (Panessa et al. 2006; Evans et al. 2006; cyan points).

The intermediate flux data are from Swift XRT (red filled points), from the Einstein IPC (data from ASDC; yellow filled points) and the observations of the Integral JEM-X from Beckmann et al. (2006, green points).

The highest flux component comes from RASS data (data from ASDC; black points), from the Einstein IPC Slew Survey (data from ASDC; yellow empty point) and from the BeppoSAX WFC (data from ASDC; magenta points). Note that the BeppoSAX WFC data with a spatial resolution of $\sim 5$ arcmin do not resolve the galaxy in X-rays and are likely contaminated by the underlying cluster emission; the same probably occurs also with the IPC Slew Survey data and with the RASS data. Note also that Integral JEM-X has a spatial resolution of $\approx 3.5 \mathrm{arcmin}$ that does not allow to resolve the central galaxy.

In the hard X-ray band, there are data obtained from two different sets of Integral ISGRI observations: the 2006 observation (Beckmann et al. 2006, green points) that have a rising spectral shape which well matches with the low-stage component seen in the soft X-rays, and the 2007 observation (Bird et al. 2007; green points), that have a spectral shape which well matches the intermediate-stage spectral component seen in the soft X-rays.

The SEDs relative to the blobs 1 and 3 of our CM model for NGC 1275 reproduce very well the X-ray data in the low and intermediate stages, while this model is not able to reproduce the X-ray data in the highest stage (i.e. the BeppoSAX WFC, RASS and Einstein IPC Slew Survey data). These last data could either indicate the presence of a further component in the SED of NGC 1275 or, more likely (as we will discuss in the next Section), are contaminated by the unresolved contribution from the thermal bremsstrahlung emission coming form the intra-cluster gas of the Perseus cluster.

\section{The diffuse emission of the Perseus cluster}

We describe in this Section the diffuse emission of the Perseus cluster atmosphere as produced by the combination of thermal bremsstrahlung from the intracluster gas and non-thermal emission from two models, i.e. the WR model and a dark matter (DM) annihilation model. We also discuss the case of a pure leptonic model at the end of this section.

\subsection{The WR model in Perseus cluster}

The WR model (see Colafrancesco \& Marchegiani 2008; Colafrancesco et al. 2004, for details) assumes the presence of cosmic ray protons in the atmosphere of the Perseus cluster and that these protons are responsible for all the heating necessary to quench the central cooling flow.

The high energy WR protons can have different origins as has been discussed in the past: they can be injected by AGN 
jets penetrating the cluster atmosphere where they further diffuse and equilibrate (see Colafrancesco \& Marchegiani 2008, for a discussion); they can be accelerated by accretion and/or merging shocks and by intracluster medium (MHD) turbulence (e.g., Colafrancesco \& Blasi 1998; Blasi \& Colafrancesco 1999; Brunetti et al. 2009; Wolfe et al. 2008); they can be accelerated by supernova remnants (Voelk et al. 1996) or by galactic cannonballs (Colafrancesco et al. 2004) occurring in cluster galaxies and then diffusing and equilibrating in the cluster atmosphere.

The assumption that WR provide all the non-gravitational heating required to quench cooling flows maximizes the amount and the role of WR protons in Perseus. However, this provides, clearly, an extreme case if other heating mechanisms (like, e.g., AGN jets and lobes, pressure waves, buoyant bubbles and cavities, intra-cluster shock waves, thermal conduction, leptonic and hadronic cosmic-rays) are at work in the same place of Perseus. For this reason, the results we present here for the case of the WR model can be considered as an upper limit case, even though extremely useful to set constraints to this model and to the amount of diffuse gamma-ray emission due to the Perseus cluster.

In the WR model that we work out here, we assume a spherical symmetry for the aims of the present discussion. In such a theoretical description, we compare our predictions with the azimuthal averaged density and temperature profiles derived from X-ray observations. In such a context, the WR model allows to obtain quantitative results by using an analytical description that retains all the essential features of the Perseus cluster structure.

It is, nonetheless, clear that in many clusters (and in particular in Perseus) we observe specific temperature and density structures that do not follow a spherical symmetry, therefore showing the complex combination of spatial regions in which heating mechanisms are more efficient and others in which cooling dominates (e.g. Fabian et al. 2003b).

The fine-grained description of these effects requires, however, a detailed numerical treatment that goes far beyond the scopes of our work.

The proton spectrum that we use for WRs in Perseus is

$N_{\mathrm{WR}}(E, r)=N_{\mathrm{WR}, 0}(E / \mathrm{GeV})^{-s}\left[g_{\mathrm{th}}(r)\right]^{\alpha}$,

with $E_{\min }=m_{\mathrm{p}} c^{2} \cdot\left[1+3.4 \times 10^{-5}(\mathrm{kT} / \mathrm{keV})\right]$ (see, e.g. Furlanetto $\&$ Loeb 2002) and $E_{\max } \rightarrow \infty$.

The spectral index $s$ is typically in the range 2.3-3.3 (see discussion in Marchegiani et al. 2007). For the specific case of Perseus, the available measurements of the mini radio halo spectrum indicate a radio (synchrotron) spectral index $\alpha_{R} \sim 1.3-1.4$ (Gitti et al. 2002) that, hence, suggest to choose a proton spectrum index $s=2.7$.

The function $g_{\mathrm{th}}(r)$ describes the radial profile of the IC gas. Churazov et al. (2003) provided a fitting formula that describes quite well both the IC gas density and the IC gas temperature outside the galaxy NGC 1275 and out to the radius $R \sim 215 h_{70}^{-1} \mathrm{kpc}$. Since this radius is of the same order of magnitude of both the cluster core radius ( $200 \mathrm{kpc}$; Churazov et al. 2003) and of the radius of the mini radio halo of Perseus ( 300 kpc; Burns 1990; Gitti et al. 2002), we assume here this value as the radius of the cluster emission region.

In the WR model the density $N_{\mathrm{WR}, 0}$ and the exponent $\alpha$ that fixes the radial distribution of non-thermal protons are determined from the request to reproduce the observed distribution of the cluster temperature by balancing, at each radius, the IC gas bremsstrahlung cooling and the heating produced by the WRs mainly through Coulomb and hadronic interactions (see details in Colafrancesco \& Marchegiani 2008; and Colafrancesco et al. 2004). The values we obtain for Perseus

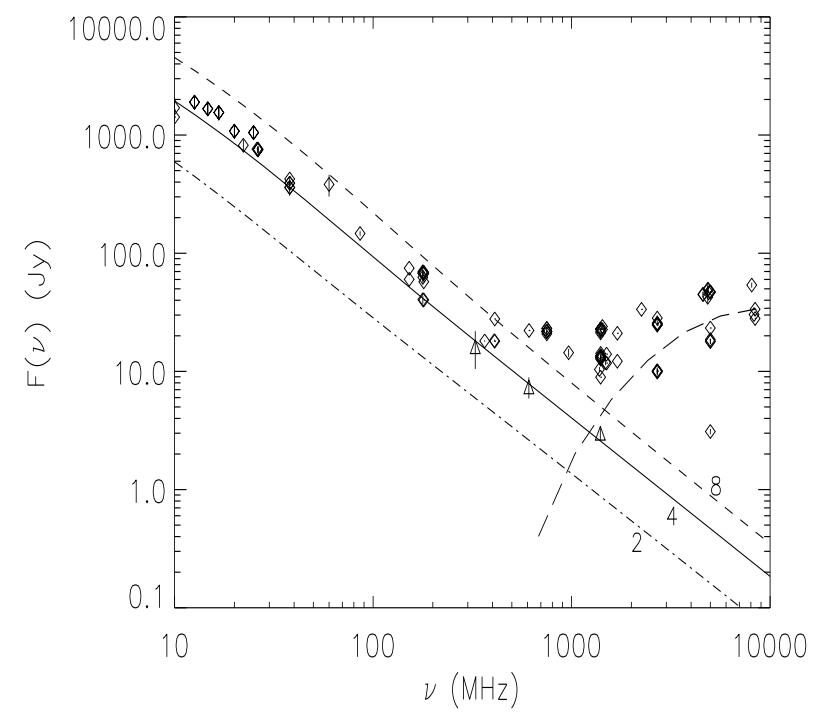

Fig. 3. Radio emission from secondary electrons in the WR model with a uniform magnetic field of 2, 4 and $8 \mu \mathrm{G}$. Long dashed curve is the SSC model for the galaxy NGC 1275 (component 1 in Table 1). Data are from Gitti et al. (2002) (triangles) and from NED (diamonds).

are: $N_{\mathrm{WR}, 0}=1.1 \times 10^{-7} \mathrm{GeV}^{-1} \mathrm{~cm}^{-3}$ (that corresponds to a pressure ratio $P_{\mathrm{WR}} / P_{\text {th }} \sim 0.54$ at the cluster center) and $\alpha=0.91$.

Figure 3 shows the radio synchrotron spectrum produced by secondary electrons in the WR model for a few values of the magnetic field (here we assume a uniform magnetic field). Figure 4 shows the comparison between the best-fitting curve of the radio brightness at $1.4 \mathrm{GHz}$ found by Pfrommer \& Enßlin (2004) with the brightness profile obtained in the WR model for a uniform magnetic field profile. We can conclude that a WR model with a uniform magnetic field of $\sim 4 \mu \mathrm{G}$ is in quite good agreement with the observed radio brightness profile of Perseus.

Figure 5 shows the ICS-on-CMB HXR emission and the non-thermal bremsstrahlung emission of the secondary electrons in the WR model compared to the thermal bremsstrahlung emission of the IC gas in the core of Perseus. The dominant nonthermal emission is given by ICS-on-CMB and it overcomes even the thermal bremsstrahlung emission at $E>40 \mathrm{keV}$. The $\mathrm{X}$-ray flux produced by ICS-on-CMB emission in the energy band $2-10 \mathrm{keV}$ is $1.5 \times 10^{-13} \mathrm{erg} \mathrm{cm}^{-2} \mathrm{~s}^{-1}$, while the flux attributed to the non-thermal component by the analysis of Sanders et al. (2005) is $6.3 \times 10^{-11} \mathrm{erg} \mathrm{cm}^{-2} \mathrm{~s}^{-1}$; it is clear that such an observed emission cannot be provided by the WR model. We must notice here (as already stressed in Sect. 2) that the result of Sanders et al. (2005) has been not confirmed by other independent observations (Molendi \& Gastaldello 2009; Eckert \& Paltani 2009; Ajello et al. 2009).

Figure 6 shows the gamma-ray emission from the Perseus cluster core as produced in the WR model. This gamma-ray emission is the sum of three different components: neutral pion decay, ICS-on-CMB ICS emission and bremsstrahlung emission both from secondary electrons. The dominant component at $E>100 \mathrm{MeV}$ is the one due to $\pi^{0} \rightarrow \gamma \gamma$ decay, and the total flux is $F(>100 \mathrm{MeV})=2.2 \times 10^{-8} \mathrm{~cm}^{-2} \mathrm{~s}^{-1}$, still lower than the EGRET upper limit on Perseus, $F(>100 \mathrm{MeV})<$ $3.72 \times 10^{-8} \mathrm{~cm}^{-2} \mathrm{~s}^{-1}$ (Reimer et al. 2003). 


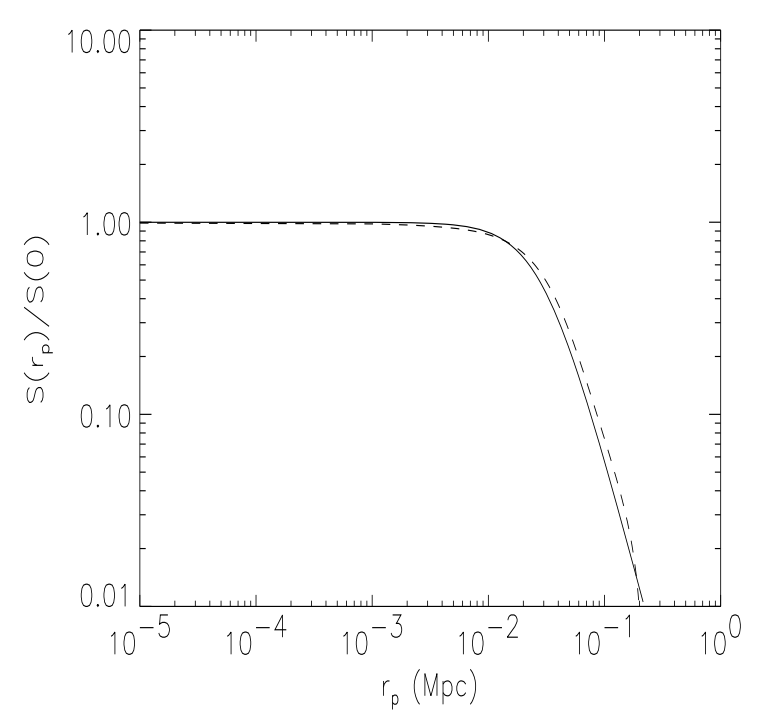

Fig. 4. Radial profile of the synchrotron radio emission from secondary electrons in the WR model (dashed curve) compared to the best-fit model of the radio-halo brightness profile at $1.4 \mathrm{GHz}$ derived from Pfrommer \& Enßlin (2004) (solid curve).

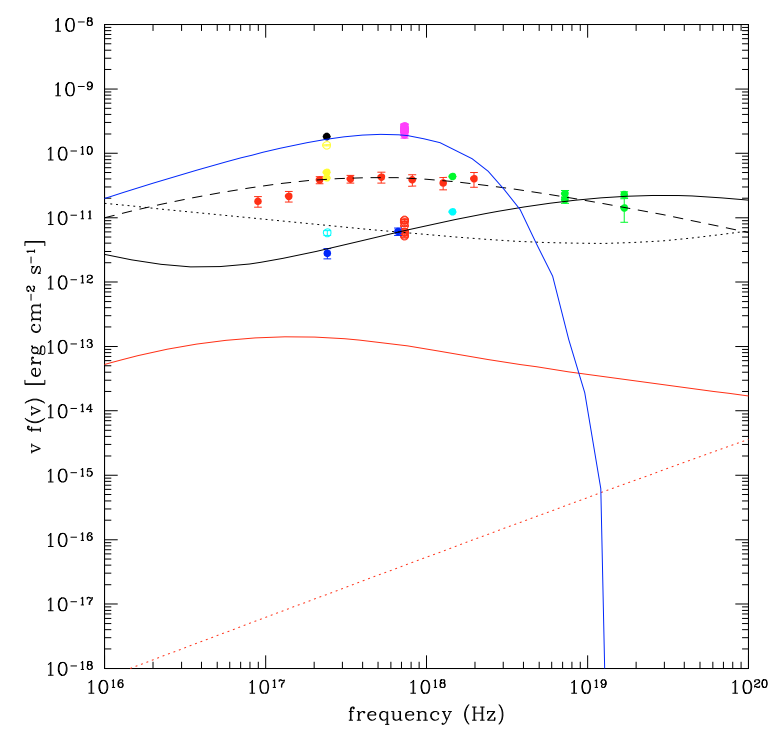

Fig. 5. The HXR ICS-on-CMB emission (red solid curve) and bremsstrahlung emission (red dotted curve) of secondary electrons in the WR model are compared to the thermal bremsstrahlung emission of the IC gas (blue solid curve) and to the three separate components of the SSC model for NGC 1275 (see Table 1): component 1 (black solid curve), 2 (dotted curve) and 3 (dashed curve). Data are taken from NED (see caption of Fig. 2 for details).

\subsection{DM models}

We consider here three neutralino DM models with neutralino masses $M_{\chi}=81\left(W^{+} W^{-}\right), 40(b \bar{b})$ and $10\left(\tau^{+} \tau^{-}\right) \mathrm{GeV}$, as we already worked out for the analysis of other clusters (see Colafrancesco \& Marchegiani 2009).

For each neutralino model we consider a radial DM density profile as given by

$g_{\mathrm{DM}}(r)=\exp \left[-(2 / \alpha)\left(\left(r / r_{\mathrm{c}}\right)^{\alpha}-1\right)\right]$

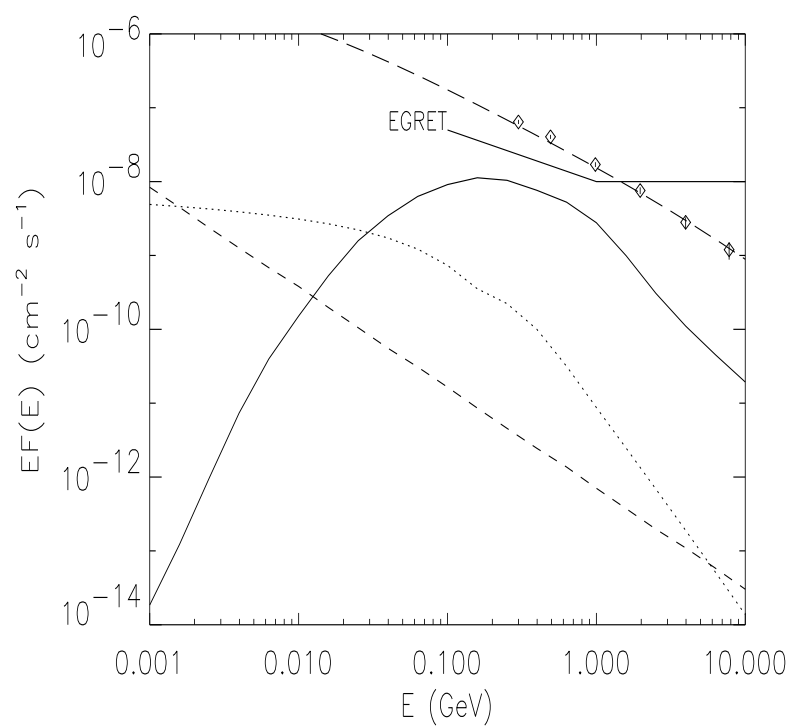

Fig. 6. The gamma-ray emission expected in the WR model from the $\pi^{0} \rightarrow \gamma \gamma$ (solid line) and from secondary electrons via ICS-on-CMB emission (short dashes line) and non-thermal bremsstrahlung (dotted line). The long-dashes line is the SSC model for NGC 1275 (component 2 in Table 1). Fermi data are from Abdo et al. (2009), while the EGRET sensitivity curve corresponds a sensitivity limit of $5 \sigma$ in 1 year of observation.

(Navarro et al. 2004), with $\alpha=0.17$ and $r_{\mathrm{c}}$ equal to the core radius of $200 \mathrm{kpc}$ (the larger core radius of the thermal gas density distribution found by Churazov et al. 2003). We assume that this DM radial profile extends out to the same region used in the WR model, $R \sim 0.215 \mathrm{Mpc}$. The spectrum of the DM source function for the secondary electrons has, consequently, a radial distribution $\propto g_{\mathrm{DM}}^{2}(r)$.

To derive the equilibrium spectrum of these secondary electrons in Perseus we consider the role of the dominant energy loss mechanisms. These are ICS losses against CMB photons and synchrotron losses for electrons with energy larger than a few hundreds $\mathrm{MeV}$ (notice that synchrotron losses for magnetic fields less than $3 \mu \mathrm{G}$, are negligible with respect to the ICS losses), while at low energies ( $\$ 150 \mathrm{MeV})$ the dominant energy loss mechanisms are Coulombian interactions with the IC gas particles. For this reason the final spatial distribution of secondary electrons is proportional to $g_{\mathrm{DM}}^{2}(r)$ at high energies $(>150 \mathrm{MeV})$ and proportional to $g_{\mathrm{DM}}^{2}(r) / n_{\mathrm{th}}(r)$ at low energies $(<150 \mathrm{MeV})$.

If the magnetic field is larger than $\sim 3 \mu \mathrm{G}$, then the dominant energy loss mechanism at high energy is synchrotron emission and, consequently, the radial distribution of the secondary electron equilibrium spectrum is $\propto g_{\mathrm{DM}}^{2}(r) / g_{B}^{2}(r)$, where $g_{B}(r)$ is the radial distribution of the magnetic field intensity.

The value of the DM annihilation cross-section directly determines the normalization of the pions and of the secondary electron density and hence of the gamma-ray emission. Since the value of this cross-section is not know, we fix it by setting the value of the heating rate due to secondary electrons compared to the value of the bremsstrahlung cooling of the IC gas in Perseus. Specifically, we used two different criteria:

i) the local heating rate is equal to the local cooling rate in the center of the cluster, e.g. at $r=10^{-6} \mathrm{Mpc}$; 


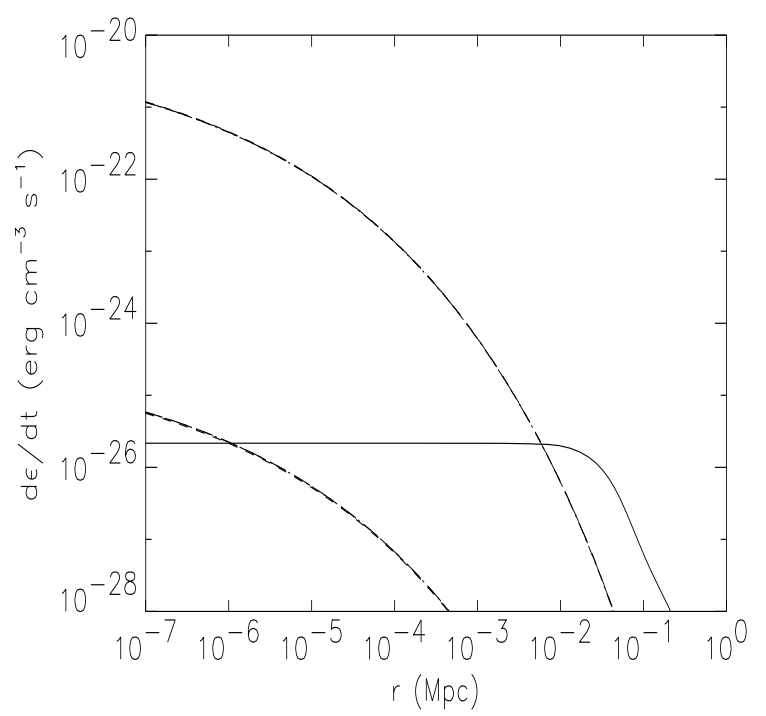

Fig. 7. The heating rate induced by DM-produced secondary electrons with a normalization given by the condition that i) the heating rate and the cooling rate equal at $r=10^{-6} \mathrm{Mpc}$ (lower curves), and ii) that their volume integral within $r=10^{-2}$ is equal (upper curves). We show different DM models with $M_{\chi}=81 \mathrm{GeV}$ (long-dashes curves), $40 \mathrm{GeV}$ (short dashes), and $10 \mathrm{GeV}$ (dot-dashes curves); continuous curve represents the IC gas cooling due to thermal bremsstrahlung.

ii) the heating rate, integrated in the volume within $r=$ $10^{-2} \mathrm{Mpc}$, is equal to the cooling rate integrated in the same region.

Figure 7 shows the comparison between the cooling rate and the heating rate due to DM annihilation as normalized according to the previous methods. The normalization method ii) yields a normalization of the secondary particle density and of the gammaray emission larger by a factor $\sim 10^{4}$. As we already noticed in Sect. 4.1 for the WR model, there can be various heating mechanisms at work in the cluster atmosphere. Therefore, the density of relativistic particles produced in DM annihilation (whose value depends, for a fixed neutralino mass, on the DM annihilation cross-section) that is set by our analysis can be considered as an upper limit. We also stress that, as in the WR model, we assume a spherically symmetric radial distribution that produces a heating rate that has a regular spherical symmetry. As discussed in Sect. 4.1, the results of our analysis can be considered as a reasonable approximation of the overall cluster physical mechanisms.

Figure 8 shows the comparison between the diffuse radio emission due to DM-produced secondary electrons and due to the secondary electrons in WR models (see Sect. 2 above) assuming the same IC magnetic field ( $B=4 \mu \mathrm{G}$ costant). The radio halo flux obtained in WR models is intermediate between the fluxes produced in the DM models with the two different normalization condition used here.

Figure 9 shows the radio halo brightness profile in DM models considered here compared to the best-fit of the Perseus radio halo brightness profile derived by Pfrommer \& Enßlin (2004): it is clear that the radio halo brightness profile produced only by the smooth DM component is much more concentrated than the observed brightness profile. Such disagreement could be solved by assuming either a profile of the magnetic field that increases towards the outer regions of the cluster (a quite unlikely solution,

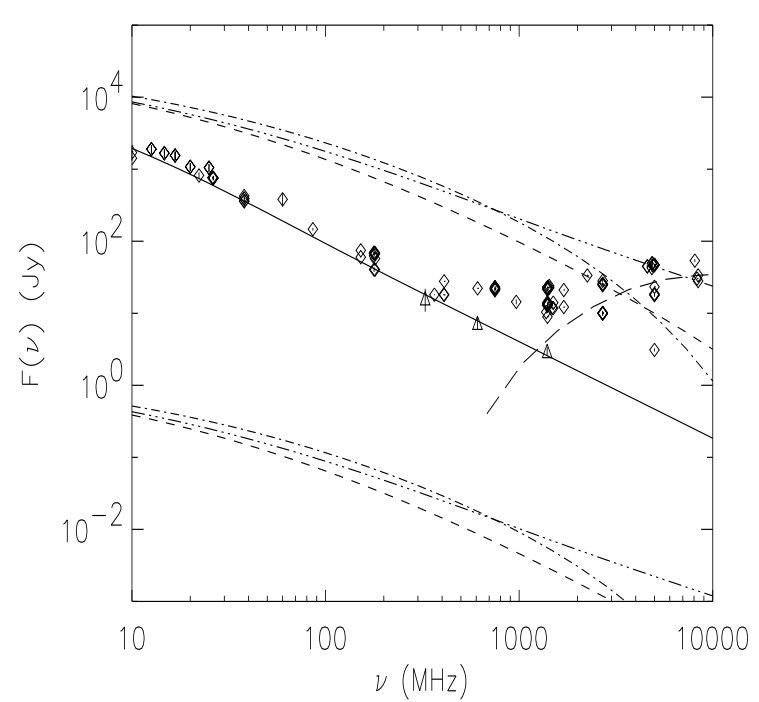

Fig. 8. Radio halo emission from secondary electrons produced from $\mathrm{DM}$ annihilation for a uniform magnetic field of $4 \mu \mathrm{G}$ and normalization fixed by the condition that the electron heating rate is equal to the cooling rate at $r=10^{-6} \mathrm{Mpc}$ (lower curves) or in the volume within $r=10^{-2} \mathrm{Mpc}$ (upper curves). We show predictions for the three DM models with $M_{\chi}=81 \mathrm{GeV}$ (three dots - dashes), $40 \mathrm{GeV}$ (short dashes) and $10 \mathrm{GeV}$ (dot-dashes); the solid thick curve shows the radio emission expected in the WR model. We also show the SSC model for NGC 1275 (lomg dashes; component 1 in Table 1). Data are from Gitti et al. (2002) (triangles) and from NED (diamonds).

as discussed by Colafrancesco et al. 2005) or, more likely, by the presence of DM substructures that can provide a larger radio brightness in the outer parts of the cluster (as discussed extensively by Colafrancesco et al. 2006).

In Fig. 10 we compare the X-ray spectrum of the thermal IC gas with the spectra produced by ICS-on-CMB in the WR model and in DM models. The flux in the $2-10 \mathrm{keV}$ produced by ICSon-CMB in the three DM models, and for the highest normalization level here considered, are $1.6 \times 10^{-12}, 1.4 \times 10^{-12}$ and $2.0 \times 10^{-12} \mathrm{erg} \mathrm{cm}^{-2} \mathrm{~s}^{-1}$, for $M_{\chi}=81,40$ and $10 \mathrm{GeV}$, respectively. In all cases, these fluxes are lower than the flux derived by Sanders et al. (2005) that is $6.3 \times 10^{-11} \mathrm{erg} \mathrm{cm}^{-2} \mathrm{~s}^{-1}$.

The different components of gamma-ray spectra (i.e. the $\pi^{0} \rightarrow \gamma \gamma$, ICS-on-CMB emission and bremsstrahlung from secondary electrons) expected from the DM models and from the WR model are shown in Fig. 11. The gamma-ray emission from both DM models with the highest normalization and from the WR model are below (a factor 3 to 10 from $300 \mathrm{MeV}$ to $8 \mathrm{GeV}$ ) the gamma-ray emission detected by Fermi towards NGC 1275 and below the EGRET upper limit (see Figs. 11 and 13). These results are consistent with the findings of the first preliminary associations of galaxy clusters with unidentified EGRET sources (Colafrancesco 2002). We note that the spectra shown are within reach of the Fermi sensitivity with integration over long exposure times of $\sim 5$ years. The gamma-ray spectra predicted in DM models with the lowest normalization here considered are instead much below (a factor $\sim 10^{3}$ ) the 1 yr Fermi sensitivity and therefore hardly detectable in the next future.

\subsection{Pure leptonic model}

We want to explore now the consequences of the presence of a diffuse population of non-thermal relativistic electrons in the 


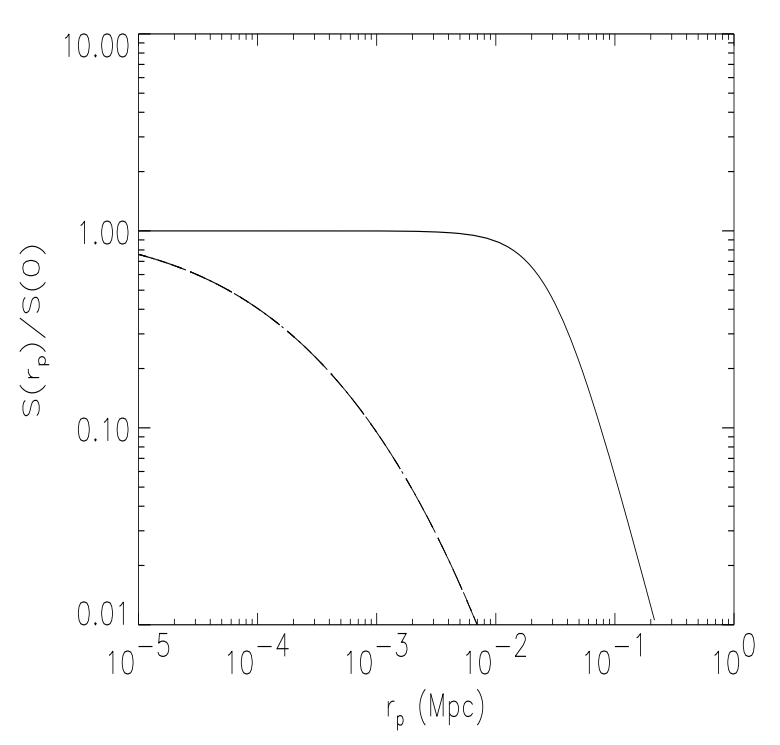

Fig. 9. Brightness profile of the radio halo produced by secondary electrons in DM models under normalization of the heating rate at the cluster center. We show the predictions of DM models with $M_{\chi}=81 \mathrm{GeV}$ (log dashes), $40 \mathrm{GeV}$ (short dashes) and $10 \mathrm{GeV}$ (dot-dashes); solid curve is the best-fit to the radio halo brightness at $1.4 \mathrm{GHz}$ found by Pfrommer \& Enßlin (2004).

atmosphere of the Perseus cluster without making assumptions on their origin. We assume specifically a relativistic electron distribution with a power-law spectrum $N_{\mathrm{e}} \propto E^{-p}$, with $p=3.7$ such that the spectral shape of the mini radio halo in Perseus can be reproduced, and we normalize the density of these electrons in order to fit the EGRET gamma-ray upper limit. This assumption is equivalent to find the maximum density of non-thermal electrons allowed by the gamma-ray upper limit measured by EGRET, and therefore such case allows us to determine the upper limit on the Hard X-Ray emission and in turn, combining the radio and HXR data, the minimum value of the magnetic field of the Perseus atmosphere. As for the normalization of the electron spectrum, we considered two different cases:

i) the electron density is normalized in such a way to reproduce the EGRET gamma-ray upper limit with their nonthermal bremsstrahlung emission. This condition implies that the electron spectrum must have a break at an energy $E \sim 80 \mathrm{MeV}$ in order to have a heating rate smaller than the thermal bremsstrahlung cooling at the cluster center. With such a spectrum, the gamma-ray emission produced by these electrons is dominated up to $E \sim 4 \mathrm{GeV}$ by the bremsstrahlung emission component. The multi-frequency behaviour of this electron spectrum implies that in the 2-10 keV band the relativistic electrons produce an ICS-onCMB flux of $6.6 \times 10^{-12} \mathrm{erg} \mathrm{cm}^{-2} \mathrm{~s}^{-1}$, smaller than the flux $6.3 \times 10^{-11} \mathrm{erg} \mathrm{cm}^{-2} \mathrm{~s}^{-1}$ estimated by Sanders et al. (2005) from the center of Perseus. The same electron population might reproduce the mini radio halo flux of Perseus assuming a central magnetic field $B_{0} \sim 3 \mu \mathrm{G}$ under the assumption that the radial profile of the magnetic field is similar to that of the intracluster gas;

ii) the electron density is normalized in such a way to reproduce the EGRET gamma-ray upper limit with their ICS-onCMB emission: in this case, the required electron density is $\sim 60$ times larger than in the previous case and, consequently,

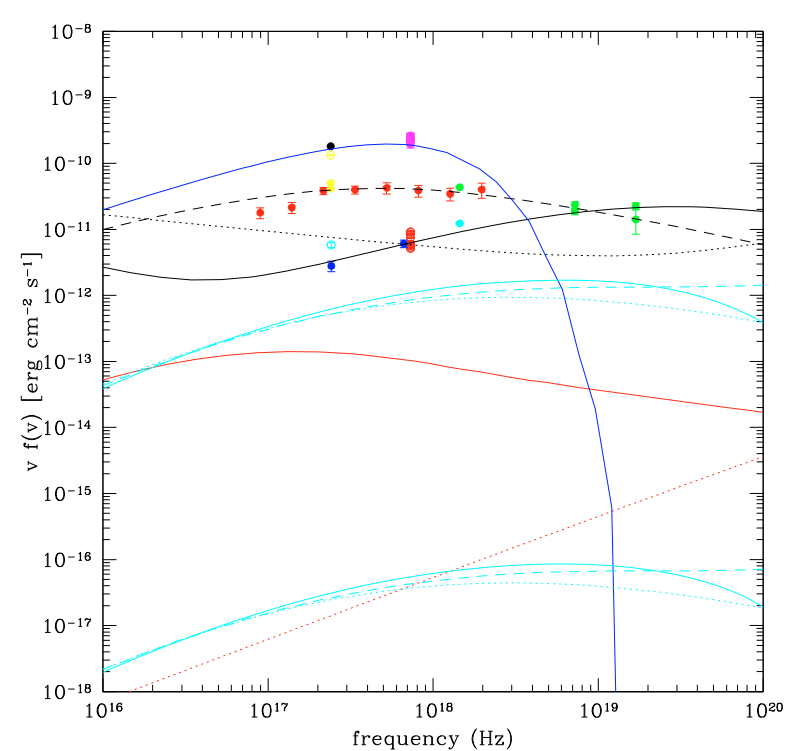

Fig. 10. HXR ICS-on-CMB emission from secondary electrons produced by DM with normalization set by the condition to have the local heating rate equal to the cooling rate at $r=10^{-6} \mathrm{Mpc}$ (lower cyan curves) or the integrated ones equal within $r=10^{-2} \mathrm{Mpc}$ (upper cyan curves). Predictions are for DM models with $M_{\chi}=81 \mathrm{GeV}$ (dashes), $40 \mathrm{GeV}$ (dotted) and $10 \mathrm{GeV}$ (solid); the red curves show the HXR ICS (solid) and bremsstrahlung (dotted) emission predicted in the WR model, while the blue solid curve shows the thermal bremsstrahlung emission from the cluster. The components 1 (black solid), 2 (black dotted) and 3 (black dashed) of the SSC model for NGC 1275 are also shown for comparison (see Table 1 for details). Data are from NED (see caption of Fig. 2 for details).

the energy scale of the spectral break required to avoid an excessive heating of the thermal gas is $E \sim 370 \mathrm{MeV}$. With such a spectrum, the gamma-ray flux is still dominated by the bremsstrahlung component up to $E \sim 4 \mathrm{GeV}$, and therefore the model in untenable because the total gamma-ray emission (bremsstrahlung plus ICS-on-CMB) would be larger than that detected by Fermi. The same conclusion is reinforced by the predictions of the multi-frequency behaviour of such electron spectrum: the ICS flux in the $2-10 \mathrm{keV}$ band is $4.2 \times 10^{-10} \mathrm{erg} \mathrm{cm}^{-2} \mathrm{~s}^{-1}$, larger than that measured, and the central value of the magnetic field required to fit the mini radio halo spectrum is $\sim 0.5 \mu \mathrm{G}$, much lower than the values usually derived for cool core clusters.

In conclusion, the density of the relativistic electrons must be normalized using their non-thermal bremsstrahlung emission, since this emission dominates on the ICS-on-CMB one. This normalization implies a lower value of the central magnetic field of $B_{0} \sim 3 \mu \mathrm{G}$ and a lower energy break at $E \sim 80 \mathrm{MeV}$ in the electron spectrum.

\section{The multi- - SED of NGC 1275 and Perseus: a comparison}

Figure 12 shows the three SSC components for NGC 1275 compared to the multi- $v$ SED of the diffuse emission of Perseus in the WR model. It is interesting to note that the diffuse cluster emission is stronger than that of the central radio galaxy only at very low radio frequency $(\$ 1 \mathrm{GHz})$, consistently with the turn over of the radio data and with the change in their spectral slope, 


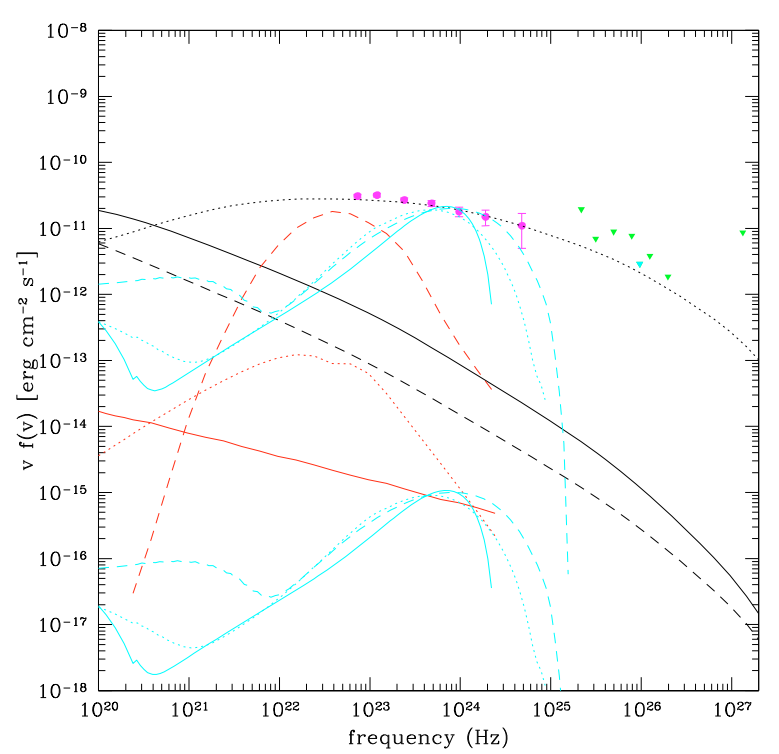

Fig. 11. The Multiple-component SED of NGC 1275 in the gammaray frequency range. The three SED components have parameters listed in Table 1: components 1 (black solid), 2 (black dotted) and 3 (black dashed). The cluster gamma-ray emission components are also shown (see caption of Fig. 10 for details). Data are from Fermi (red points) and the upper limits are from MAGIC (green) and WHIPPLE (cyan).

and differently from the behaviour of the SSC model for the nucleus of NGC 1275 and of AGNs in general (see Colafrancesco \& Giommi 2006). It is important to notice that at such low frequency radio measurements have a lower angular resolution that does not allow to distinguish between the galaxy emission and the diffuse cluster emission.

Figure 11 shows the comparison between the gamma-ray part of the SED of NGC 1275 and the gamma-ray emission of the Perseus cluster.

The cluster SED, both in the WR model and in DM models, are significantly lower and with significantly different spectral shape with respect to the SED of NGC 1275 and to the Fermi data.

The WR model predicts a flux of $2.2 \times 10^{-8} \mathrm{~cm}^{-2} \mathrm{~s}^{-1}$, almost one order of magnitude lower than the NGC 1275 flux observed with Fermi. In order to reproduce the Fermi data, a density of relativistic protons 10 times higher would be necessary in the WR model, but such high proton density would provide an excessive heating of the intra-cluster gas.

The DM models with their highest mormalization could reach the Fermi flux value but could not reproduce the Fermi spectrum. We also stress that this normalization produces an excessive heating rate at the cluster center (see Fig. 7).

The Fermi data are very well fitted in a CM model for blazars and radio galaxies (Colafrancesco et al. 2010, in prep.) with three components for NGC 1275, in which the blob n.2 (see parameters in Table 1) produces all of the emission observed at gamma-ray energies, while it produces a sub-dominant emission in the other frequency ranges, apart from the extreme UV and soft gamma-rays (see Fig. 12). This SED is also consistent with the existing TeV limits. The gamma-ray historical variability (see the Fermi and EGRET data) is also consistent with a multiple blob scenario in the CM model and excludes also that the bulk of gamma-ray emission is due to the diffuse emission from the cluster atmosphere.



Fig. 12. The three SSC components for NGC 1275 as in Fig. 1 are compared with the various diffuse emission components (as labelled) of the Perseus cluster in the WR model (solid curves). Data from Gitti et al. (2002) (triangles) are added w.r.t. Fig. 1.

In the X-ray frequency range, (see Fig. 10) the various nonthermal emissions from the cluster are always lower than the observations obtained with different instruments. The thermal bremsstrahlung from the cluster is instead clearly observed and is consistent with the ROSAT and BeppoSAX WFC observations. The CM model with three components for NGC 1275 is instead able to reproduce all other X-ray observations with higher angular resolution and the historical variability of the source clearly seen at these frequencies.

Our results indicate that the frequency ranges in which the diffuse cluster SED is detected, and exceeds the SED of NGC 1275, are those of the low-frequency radio range $v \lesssim$ $1 \mathrm{GHz}$ and that of the soft X-rays with $E \sim \mathrm{keV}$. The SED observed in all other frequency ranges is dominated by the central galaxy NGC 1275 . The existing data are best explained by a CM model with three main components in which the first one produces most of the emission observed from radio to optical frequencies and the lowest state of the X-ray emission, the second one produces most of the gamma-ray emission and the third one produces the higher state of the X-ray observations.

\section{Discussion and conclusions}

In this paper we studied the gamma-ray emission features of the combined system of the NGC 1275 galaxy living in the core of the Perseus cluster based on a multi-component model and in multi-frequency approach. This study has been done with the aim to assess a strategy that is able to disentangle the gamma-ray emission of the cluster from that of the galaxy NGC 1275.

Our analysis provides various constraints on the NGC 1275 emission properties, on the cluster atmosphere, and on the interaction of the two sources.

We worked out a CM model for NGC 1275 that is able to reproduce the Fermi observations, the yearly (or monthly) variability of this source in gamma-rays and X-rays, and the different observed intensity states of its multi-frequency SED (see Figs. 1 and 2). We find that the Fermi detection of NGC 1275 is entirely 
due to the emission of a compact $\left(r \sim 8 \times 10^{-3} \mathrm{pc}\right)$ and energetic blob filled with an electron population with double power law spectrum with $\gamma_{\mathrm{b}} \approx 5 \times 10^{3}$, with $\delta \approx 8$ and relatitely high normalization factor $N \approx 6.8 \mathrm{~cm}^{-3}$ (see Table 1). The SED of this blob perfectly fits the Fermi spectrum and is also consistent with MAGIC and WHIPPLE upper limit at TeV energies. We also note that the upper limit obtained by VERITAS (Acciari et al. 2009) is lower than the flux of the high-energy component of the CM model (see Fig. 14). We notice that Acciari et al. (2009) actually calculated integral upper limits at $E>400 \mathrm{GeV}$, for which the transformation to SED upper limits requires assumptions that might introduce further uncertainties. However, Acciari et al. (2009) pointed that the Fermi flux measurements simultaneous with the VERITAS ones are lower by a factor of $\sim 1.37$ w.r.t. the data of Abdo et al. (2009). Therefore, the VERITAS upper limits are consistent with the fact that they have been measured while the central source was decreasing its high-energy flux, and are hence completely consistent with a variable high-E component of the NGC 1275 SED as predicted by the CM model.

For the surrounding Perseus cluster atmosphere, DM annihilation models with high normalization are excluded because they produce a stationary gamma-ray flux of the same order of that observed at $E \sim 3 \mathrm{GeV}$ by Fermi from NGC 1275, and - in addition - they produce a spectrum of gamma-ray emission that is strongly peaked at a few $\mathrm{GeV}$, contrary to the spectral shape of the gamma-ray source detected by Fermi (see Fig. 11).

The WR model produces instead a gamma-ray flux always much lower (by a factor of 5 to more than 10) than the gamma-ray flux from NGC 1275 observed by Fermi.

For a pure leptonic model, the EGRET upper limit sets an upper limit to the relativistic electrons density or, combining the gamma measurements with the radio ones, a lower limit to the magnetic field. We have verified that the non-thermal bremsstrahlung emission dominates on the ICS-on-CMB one. For this model, a lower value of the central magnetic field of $B_{0} \sim 3 \mu \mathrm{G}$ is found, and a lower spectral cutoff of $E \sim 80 \mathrm{MeV}$ in the electron spectrum is required by the heating constraints.

Due to the fact that the gamma-ray emission of NGC 1275 is variable, it is possible to reveal the diffuse gamma-ray emission from Perseus during a state of low activity of NGC 1275. In the core of the Perseus cluster, the diffuse gamma-ray flux at $E>100 \mathrm{MeV}$ from the cluster atmosphere (both in the WR model and in the DM annihilation model with the highest normalization, that produces an over-heating at the cluster center) could be detected by Fermi if NGC 1275 is found in a low-state as that produced by the first blob in our CM model (component 1 in Table 1, see also Fig. 13). The diffuse gamma-ray flux coming from these diffuse components might be also marginally detectable by the future CTA experiment at $E \gtrsim 10 \mathrm{GeV}$. The gamma-ray flux produced by DM annihilation with the lowest normalization would be undetectable by both Fermi and CTA being its peak flux at $\sim 1 \mathrm{GeV}$ more than a factor 300 below the Fermi $(1$ yr., $5 \sigma)$ sensitivity.

An alternative approach to disentangle the cluster gammaray emission from that of NGC 1275 is to determine the diffuse flux outside the cluster core where the emission from the central galaxy is no longer contaminating the measurements. At $E>1 \mathrm{GeV}$ the Fermi $68 \%$ collecting angle is $\sim 0.6 \mathrm{deg}$. that corresponds to $\sim 786 \mathrm{kpc}$ from the cluster center: thus Fermi can have the possibility to resolve and detect the diffuse gamma-ray flux coming from the outer corona of the Perseus cluster atmosphere at distances $r \gtrsim 800 \mathrm{kpc}$ from the center of the cluster. The gamma-ray flux from Perseus evaluated in the corona between 0.786 and $1 \mathrm{Mpc}$ (i.e., approximately the maximum radius

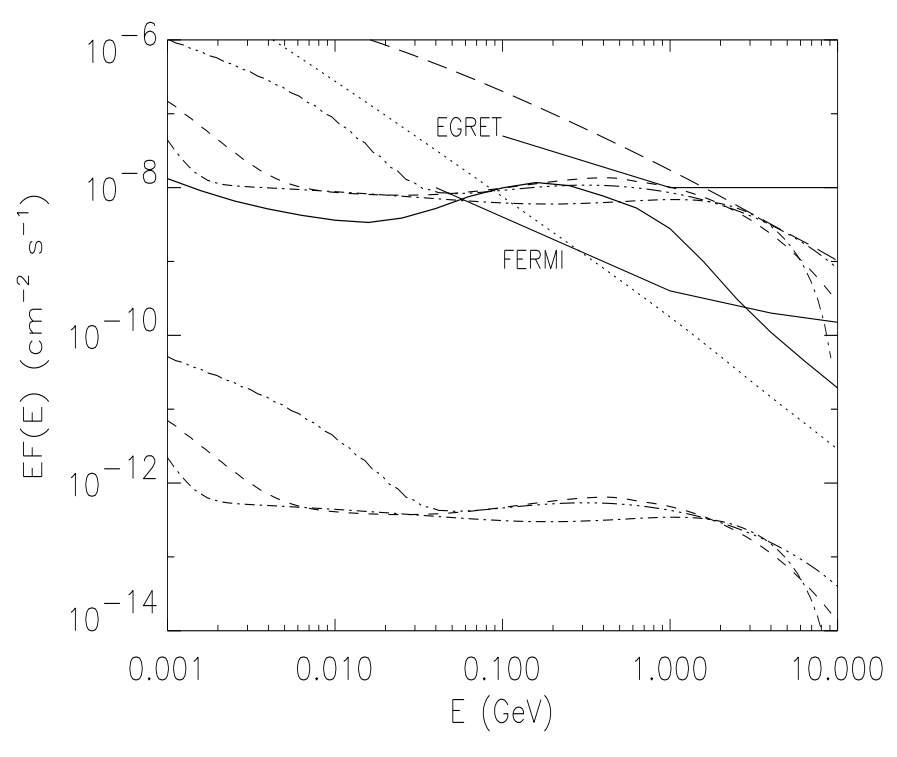

Fig. 13. Gamma-ray emission of the NGC 1275 CM model component 1 (dotted line) and 2 (long-dashed line), of cluster WR model (thick line) and DM models with $M_{\chi}=81,40$ and $10 \mathrm{GeV}$ (three dots-dashed, dashed and dot-dashed lines respectively) with high and low normalization. Sensitivity limits at $5 \sigma$ level for $1 \mathrm{yr}$ of observation for EGRET and Fermi are also shown.

to which $\mathrm{X}$-ray thermal emission is observed; see, e.g., Furusho et al. 2001) is $F(>1 \mathrm{GeV}) \approx 1.2 \times 10^{-10} \mathrm{~cm}^{-2} \mathrm{~s}^{-1}$ in WR model, which can be marginally detectable from Fermi. Therefore, such a strategy could provide a realistic case to detect the diffuse gamma-ray emission from the Perseus cluster.

This argument does not apply to the case of DM models because in such a case the gamma-ray flux comes mainly from the inner cluster region due to the $\propto n_{\mathrm{DM}}^{2}(r)$ dependence of the annihilation flux. This implies that even a spatially resolved observation of the Perseus cluster in gamma-rays cannot provide information on the DM models since the relative gamma-ray brightness is not observable at large angular distances from the central galaxy.

As for the full multi-frequency approach, we find that the diffuse emission produced in the cluster core is much less than that of the central galaxy (Fig. 12), with the exception of two frequency windows: the radio frequency region at $v \lesssim 1 \mathrm{GHz}$ (Fig. 3) in which the diffuse mini radio halo of Perseus dominates the emission, and the soft X-ray band $(E \sim \mathrm{keV})$, at which the thermal emission from the cluster gas peaks (Fig. 5). The dominance of the cluster diffuse emission in these frequency ranges is a well known result.

The WR model is the one that better fits both the mini radio halo features (requiring a magnetic field radial profile almost constant in the cluster core $R \leq 215 \mathrm{kpc}$ see Fig. 4 and with a value of $B \sim 4 \mu \mathrm{G}$, consistently with Faraday Rotation measurements, see Carilli \& Taylor 2002) and the flux and temperature distribution of the diffuse, X-ray emitting plasma. DM annihilation models produce, instead, a much steeper density profile towards the cluster center (Fig. 9), and might then reproduce the radio halo brightness profile only for a B-field radial profile which rapidly increases towards the outer cluster regions.

In summary, the gamma-ray emission from the Perseus cluster is dominated in its central region by the central radio galaxy NGC 1275 , a situation similar to those found for the first preliminary evidence found with EGRET of gamma-ray emitting 


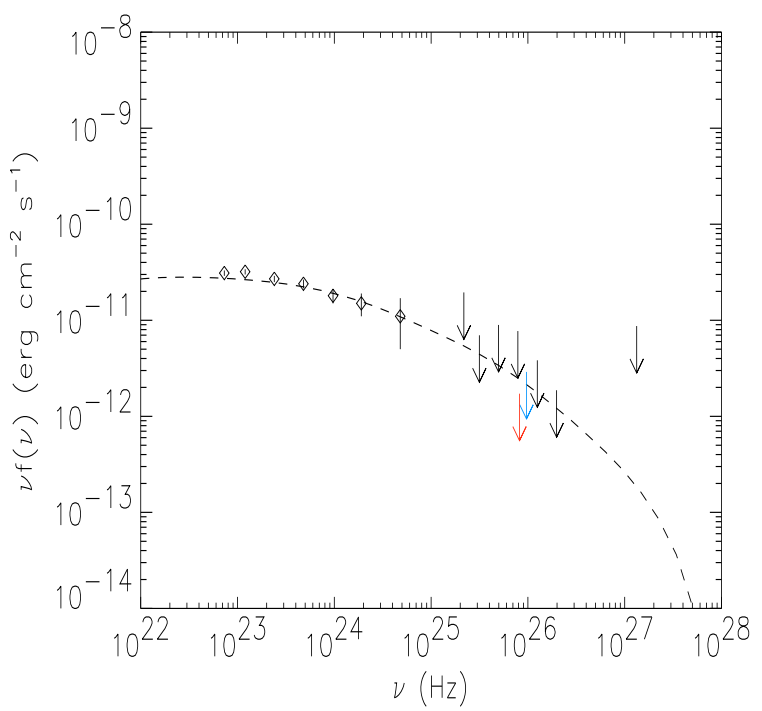

Fig. 14. A close-up of the high-energy spectral region of the SED of NGC 1275: the dashed line is the component 2 of the CM. Data are from Fermi (open circles). The upper limits are from MAGIC (black), WHIPPLE (blue) and VERITAS (red). We refer the reader to Aleksic et al. (2010) for a discussion of the comparison of the limits obtained by Cherenkov telescopes.

clusters hosting powerful radio galaxies (see the previous conclusions by Colafrancesco 2002). This seems to be the most promising case to observe galaxy cluster in gamma-rays at the moment, even though the expected detectable number of clusters is quite limited to a few specific cases. These conclusions are certainly quite different from those of other (accreting and merging cluster) models in which it has been predicted that the operating gamma-ray missions should have already discovered a significant number (i.e., 5 to 7 clusters with AGILE, and about 20 clusters with Fermi) after 1 yr. operation (see, e.g., Blasi et al. 2007).

The diffuse gamma-ray flux from the Perseus cluster can be detected in two regions: i) in the cluster core, for both DM and WR models, when the gamma-ray emission from NGC 1275 is found in a low activity state; ii) in the outer cluster region at $r \gtrsim 800 \mathrm{kpc}$ only for the WR model.

In conclusion, our results show that a simultaneous study of the various emission mechanisms that produce diffuse gamma-rays from galaxy clusters and the study of the emission mechanisms that produce gamma-rays from active galaxies residing in the cluster atmospheres is absolutely crucial first to disentangle the spectral and spatial characteristics of the gamma-ray emission and secondly to assess the optimal observational strategy in the attempt to reveal the still elusive diffuse gamma-ray emission widely predicted for the atmospheres of large-scale structures.

Acknowledgements. We acknowledge the extensive use of the multi-frequency data obtained from the ASI-ASDC and available on line from its web site http: //www . asdc . asi .it. Historical data for NGC 1275 have also been taken by the NASA/IPAC Extragalactic Database. We thank F. Zandanel for providing useful comments on Cherenkov telescope data.

\section{References}

Abdo, A. A., Ackermann, M., Ajello, M., et al. 2009, ApJ, 699, 31 Acciari, V. A., Aliu, E., Arlen, T., et al. 2009, ApJ, 706, L275
Ajello, M., Rebusco, P., Cappelluti, N., et al. 2009, ApJ, 690, 367 Aleksic, J., Pfrommer, C., Pinzke, A., et al. 2010, ApJ, 710, 634 Angel, J. R. P., \& Stockman, H. S. 1980, ARA\&A, 18, 321

Asada, K., Kameno, S., Shen, Z.-Q., et al. 2006, PASJ, 58, 261 Balmaverde, B., Capetti, A., \& Grandi, P. 2006, A\&A, 451, 35 Beckmann, V., Gehrels, N., Shrader, C. R., \& Soldi, S. 2006, ApJ, 638, 642 Bird, A. J., Malizia, A., Bazzano, A., et al. 2007, ApJS, 170, 175 Blasi, P., Gabici, S., \& Brunetti, G. 2007, Int. J. Mod. Phys., A22, 681 Boehringer, H., Voges, W., Fabian, A. C., et al. 1993, MNRAS, 264, L25 Brunetti, G. 2003, ASPC, 301, 349

Brunetti, G., Blasi, P., Cassano, R., \& Gabici, S. 2009, AIPC, 1112, 129

Burns, J. O. 1990, AJ, 99, 14

Carilli, C. L., \& Taylor, G. B. 2002, ARA\&A, 40, 319

Churazov, E., Forman, W., Jones, C., \& Böhringer, H. 2003, ApJ, 590, 225

Colafrancesco, S. 2002, A\&A, 396, 31

Colafrancesco, S. 2007, CHJAAS, 8, 61

Colafrancesco, S. 2009, in Proceedings of the Meeting High-Energy Gamma-rays and Neutrinos from Extra-Galactic Sources, Heidelberg, 13-16 Jan., in press

Colafrancesco, S., \& Blasi, P. 1998, Astropart. Phys., 9, 227

Colafrancesco, S., \& Mele, B. 2001, ApJ, 562, 24

Colafrancesco, S., \& Giommi, P. 2006, ChJAS, 6, 47

Colafrancesco, S., \& Marchegiani, P. 2008, A\&A, 484, 51

Colafrancesco, S., \& Marchegiani, P. 2009, A\&A, 502, 711

Colafrancesco, S., Dar, A., \& De Rújula, A. 2004, A\&A, 413, 441

Colafrancesco, S., Marchegiani, P., \& Perola, G. C. 2005, A\&A, 443, 1

Colafrancesco, S., Profumo, S., \& Ullio, P. 2006, A\&A, 455, 21

Colafrancesco, S., Lieu, R., Marchegiani, P., Pato, M., \& Pieri, L. 2010, A\&A, submitted [arXiv: 1004.1286]

Dennison, B. 1980, ApJ, 239, L93

Eckert, D., \& Paltani, S. 2009, A\&A, 495, 415

Evans, D. A., Worrall, D. M., Hardcastle, R. P., et al. 2006, ApJ, 642, 96

Fabian, A. C., Sanders, J. S., Allen, S. W., et al. 2003a, MNRAS, 344, L43

Fabian, A. C., Sanders, J. S., Crawford, C. S., et al. 2003b, MNRAS, 344, L48

Fabian, A. C., Sanders, J. S., Taylor, G. B., et al. 2006, MNRAS, 366, 417

Furlanetto, S. R., \& Loeb, A. 2002, ApJ, 572, 796

Furusho, T., Yamasaki, N. Y., Ohashi, T., et al. 2001, ApJ, 561, L165

Giommi, P., Colafrancesco, S., Cutini, S., et al. 2008, A\&A, 487, L49

Gitti, M., Brunetti, G., \& Setti, G. 2002, A\&A, 386, 456

Houston, B. P., Wolfendale, A. W., \& Young, E. C. M. 1984, J. Phys. G: Nucl. Phys., 10, L147

Inoue, S., Aharonian, F., \& Sugyiama 2005, ApJ, 628, L91

Kellermann, K. I., Lister, M. L., Homan, D. C., et al. 2004, ApJ, 609, 539

Kushnir, D., \& Waxman, E. 2009, JCAP, 8, 2

Lister, M. L., Aller, H. D., Aller, M. F., et al. 2009, AJ, 137, 3718

Marchegiani, P., Perola, G. C., \& Colafrancesco, S. 2007, A\&A, 465, 41

Mayer-Hasselwander, H. A., Kanbach, G., Bennett, K., et al. 1982, A\&A, 105, 164

Miniati, F., Jones, T. W., Kang, H., \& Ryu, D. 2001, ApJ, 562, 233

Molendi, S., \& Gastaldello, F. 2009, A\&A, 493, 13

Navarro, J. F., Hayashi, E., Power, C., et al. 2004, MNRAS, 349, 1039

Nevalainen, J., Oosterbroeck, T., Bonamente, M., \& Colafrancesco, S. 2004, ApJ, 608, 166

Osako, C. Y., Ulmer, M. P., Grabelsky, D. A., et al. 1994, ApJ, 435, 181

Panessa, F., Bassani, L., Cappi, M., et al. 2006, A\&A, 455, 173

Perkins, J. S., Badran, H. M., Blaylock, G., et al. 2006, ApJ, 644, 148

Pfrommer, C. 2008, MNRAS, 385, 1242

Pfrommer, C., \& Enßlin, T. A. 2004, MNRAS, 352, 76

Primini, F. A., Basinska, E., Howe, S. K., et al. 1981, ApJ, 243, L13

Pronik, I. I., Merkulova, N. I., \& Metik, L. P. 1999, A\&A, 351, 21

Reimer, O., Pohl, M., Sreekumar, P., \& Mattox, J. R. 2003, ApJ, 588, 155

Sanders, J. S., \& Fabian, A. C. 2007, MNRAS, 381, 1381

Sanders, J. S., Fabian, A. C., \& Dunn, R. J. H. 2005, MNRAS, 360, 133

Sarazin, C. L. 1999, ApJ, 520, 529

Strong, A. W., \& Bignami, G. F. 1983, ApJ, 274, 549

Strong, A. W., Bignami, G. F., Caraveo, P. A., et al. 1982, A\&A, 115, 404

Taylor, G. B., \& Vermeulen, R. C. 1996, ApJ, 457, L69

Timokhin, A. N., Aharonian, F. A., \& Neronov, A. Yu. 2004, A\&A, 417, 391

Vermeulen, R. C., Readhead, A. C. S., \& Backer, D. C. 1994, ApJ, 430, L41

Véron-Cetty, M. P., \& Véron, P. 1998, Quasars and Active Galactic Nuclei (8th edition)

Vittorini, V., Tavani, M., Paggi, A., et al. 2009, ApJ, 706, 1433

Voelk, H. J., Aharonian, F. A., \& Breitschwerdt, D. 1996, Space Sci. Rev., 75, 279

Walker, R. C., Dhawan, V., Romney, J. D., et al. 2000, ApJ, 530, 233

Wolfe, B., Melia, F., Crocker, R. M., \& Volkas, R. R. 2008, ApJ, 687, 193 